\title{
Impedance of Metal Organic Frameworks: On the Way to in-situ Monitoring of Proton Conducting Membranes
}

\author{
C. Weinberger $^{1}$, D. Klawinski ${ }^{1}$, A. Javed ${ }^{1}$, T. Homburg, ${ }^{2}$ N. Stock, ${ }^{2}$ M. Tiemann ${ }^{1}$, T. \\ Wagner ${ }^{1, *}$ \\ 1,2 University of Paderborn, Faculty of Science, Department of Chemistry, \\ Warburger Str. 100, D-33098 Paderborn, Germany \\ thorsten.wagner@upb.de
}

\begin{abstract}
:
The presented project aims at the development of an in-situ characterization method for the development of new proton conducting Metal-Organic Frameworks (MOFs). A novel combination of water sorption technique with impedance spectroscopy allows identification of proton conduction mechanisms in complex nanomaterials. On standard proton conducting materials (e.g. Nafion) the relative humidity determines the dominating conduction type (proton hopping, Grotthuss or vehicle mechanism). Discrimination is possible by temperature resolved studies, which can be utilized for optimization of specifically designed, humidity-independent proton-conducting MOFs. First results on the characterization of the novel setup as well as on single crystal MOF materials will be presented.
\end{abstract}

Key words: Impedance Spectroscopy, Metal-Organic Framework, Crystallite, PEMFC, Proton Conductivity

\section{Results}

Properties like high proton conduction and electrode insulation of polymer membranes allow them to act as a pivotal component in polymer electrolyte membrane fuel cells (PEMFs) [1,2]. Typical materials used today exhibit strong correlation of proton conductivity with operating conditions, specifically humidity. Therefore, the control of operating conditions based on empirical results already helps to improve the efficiency of PEMFCs.

Following are the two approaches that will be presented: The use of IS technique to investigate the effect of humidity on proton conduction mechanisms in MOFs with spatial resolution by using electrodes of different geometries (fringe electric field) [3].

The use of metal organic frameworks (MOFs) and coordination polymers (CPs) [4,5] as moisture-independent proton conductors. The use of suitable linkers allows manipulation of the ionic conductivity [6].

The motive was to develop a particular structure for measurement of anisotropic effects and check the influence of relative humidity and temperature. This will facilitate us with a systematic study of several MOFs.

Figure 1 displays the result of proton conduction in single crystal of novel MOF lanthanum phosphonatosulfonate (LaSSPP). The proton resistance was calculated using equivalent circuit model and effect of ambient humidity (35-80\% RH) was determined in a nitrogen stream at $23{ }^{\circ} \mathrm{C}$. The graph exhibits a significant decrease in proton resistivity at $50 \%$ adjusted relative humidity. 

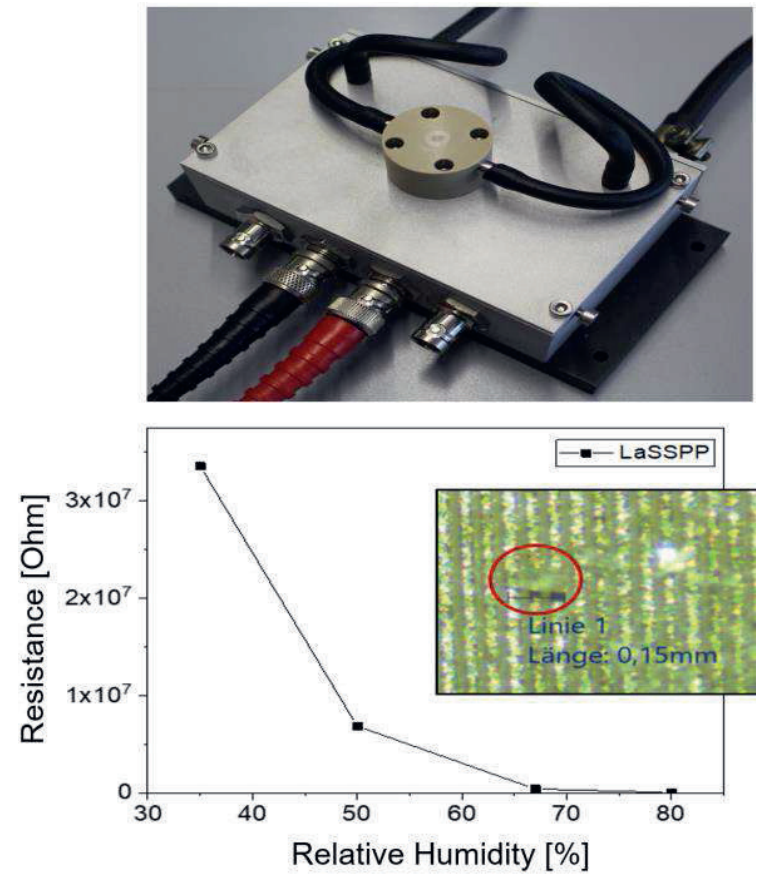

Fig. 1. Measurement setup (top) and the proton resistance of LaSSPP crystallites depending on relative humidity (bottom).

\section{References}

[1] U. Sen, S.Ü. Çelik, A. Ata, A. Bozkurt, Int. J. Hydrogen Energy 2008, 33, 2808-2815.

[2] Z. Rao, C. Zheng, F. Geng, Comput. Mater. Sci., 2018, 142, 122-128.

[3] S.M. Rezaei Niya, M. Hoorfar, J. Power Sources, 2013, 240, 281-293.

[4] H. Furukawa, K. E. Cordova, M. O’Keeffe, O. M. Yaghi, Science, 2013, 341, 1230444-1.

[5] A.-L. Li, Q. Gao, J. Xu, X.-H. Bu, Coord. Chem. Rev. 2017, 344, 54-82.

[6] O. Beyer, T. Homburg, M. Albat, N. Stock, U. Lüning, New J. Chem, 2017, 41, 8870-8876. 